\title{
Direct Reconstruction of Functional Parameters for Dynamic SPECT
}

\author{
MA Limber ${ }^{\dagger}$, MN Limber $^{\dagger}$, A Celler ${ }^{\ddagger}$, JS Barney ${ }^{\dagger}, \mathrm{JM}$ Borwein ${ }^{\dagger}$ \\ $\uparrow$ Centre for Experimental and Constructive Mathematics, Simon Fraser University, \\ Burnaby, BC, Canada, V5A 1S6 \\ $\ddagger$ Div. of Nuclear Medicine, Vancouver Hospital and Health Sciences Centre, \\ Vancouver, BC, Canada, V5Z 1M9
}

\begin{abstract}
We have developed a dynamic SPECT method which allows the prevalent rotating camera systems to be used. In this method, the parameters of the time-activity curve in each pixel are recovered directly from the projection data, without first reconstructing a series of static images. Preliminary test results show that we can accurately recover time-activity curves for a simple model based on activity values expected for myocardial viability studies with fatty acid tracers.
\end{abstract}

\section{Introduction}

The ability to obtain functional information from the living human body has powerful implications in both the clinical and research environments. SPECT (Single Photon Emission Computed Tomography) and PET (Positron Emission Tomography) are recognized as among the imaging techniques best suited to investigate function, as they are sensitive to bio-distribution of radiopharmaceuticals within the body. In many cases, it is the change in these distributions with time that offers the most information about the underlying physiological processes. The purpose of dynamic imaging is to quantify these changes.

At present, clinical dynamic studies use sequences of images (reconstructed images in the case of SPECT and PET), in which each image is intended to represent the spatial distribution of the tracer at some particular moment. In planar imaging, curve fitting or compartmental analysis techniques can be applied directly to a region of interest (ROI) in the image or on a pixel by pixel basis to obtain dynamic information (for example $[1,2,3]$ ). Tomographic techniques which study kinetics of metabolic processes, such as PET $[4,5]$ or ring camera SPECT systems $[6,7]$, acquire all projections for each image simultaneously. The images can then be reconstructed using conventional methods and counts in ROIs analysed to estimate the kinetic parameters of the dynamic model.

For reasons of availability and cost, it would be beneficial to have tomographic dynamic imaging for conventional rotating camera SPECT. Two problems arise in a direct application of conventional dynamic imaging methods to

0018-9499/95\$4.00 rotating camera SPECT. First, because of the low sensitivity of the SPECT system, there is a tradeoff between image counts and resolution in time. In order to obtain sufficient count statistics data for each image in the sequence must be collected over a relatively long time. Second, the activity distribution changes with time and the projections are not taken at the same moment in a rotating camera system. As a consequence, the projection sets used to reconstruct each of the images will not be consistent.

A recent study applied conventional image reconstruction methods to a rotating SPECT camera for dynamic analysis of IPPA metabolism in the myocardium [8]. Monoexponential functions were fit to ROI's in a sequence of five images each taken at 4,12,20, 28 and 36 minutes after injection. The time resolution in these studies is much worse than comparable planar studies, which can use four images per minute [2], and so does not allow determination of the fast element of fatty acid metabolism. In addition, the images are subject to reconstruction artifacts due to the change in activity during the data collection period.

Multi-detector systems, with up to three heads, have recently been introduced. Such systems have higher sensitivity and the ability to collect complete data sets more quickly than the prevalent single head cameras, which mitigates both problems outlined above. Exploitation of the capability of the new cameras to improve the performance of conventional dynamic imaging techniques applied to SPECT has been proposed for myocardial perfusion studies $[9,10,11]$. The method, however, remains limited by the rate of change of the radiopharmaceutical distribution relative to the time for acquisition of a complete projection set required to reconstruct an image. Also, multi-head systems are still not as widely available as single-head cameras.

An alternative approach is to estimate parameters of the dynamic model directly from the projection data, without image reconstruction. A method for directly reconstructing functional images has been described for PET [12], but the approach is sufficiently general that it can also be applied to rotating camera SPECT. Other direct reconstruction methods for tomography have focused on estimating the dynamic parameters in a specified ROI $[13,14,15]$ or, recently, on determining both the ROI boundary and the (c) 1995 IEEE 
dynamic parameters within that boundary [16].

Here we present a dynamic imaging method for direct reconstruction of the functional parameters of a simple physiological model that can be used with standard rotating SPECT cameras. The main emphasis of our study is on clinical practicality. We attempt to develop an effective SPECT technique for the particular diagnostic problem of estimating the time-activity curves for IPPA metabolism in the myocardium. A starting point is a functional representation of changes in the activity distribution in the heart which was determined from the previous studies $[2,17,18]$.

The basic question which is addressed in this paper is whether direct parameter reconstruction is a viable technique for clinical dynamic SPECT. In this respect the experimental conditions were modeled on protocols which are used in real life clinical situations. The activity values and time constants used here were based on values expected for myocardial viability studies estimated from planar studies. The simulated acquisition protocols met the restrictions of commonly used SPECT system, which can perform only a single 360 degree rotation.

The statistical noise in the data related to the limited number of counts available for the analysis is an important problem in SPECT imaging. In this respect the answer to the question of stability of parameter reconstruction method for very low and constantly decreasing count rates was considered essential and was emphasised in our study. We found that the direct reconstruction method could recover the dynamic parameters with reasonable accuracy, even from noisy data. Attenuation correction (using a known, homogeneous attenuating medium) was also tested with good results.

Our method assumes an explicit exponential functional form for the time-activity distribution for each pixel in the image. This approach is simpler than compartmental modeling, but is well justified for modeling IPPA metabolism in the myocardium by previous work in planar imaging $[17,18]$.

Another approach to direct parameter reconstruction recently proposed for SPECT estimates parameters of a compartmental model using sequential parameter estimation [19]. The compartmental model of the underlying dynamic function is more general than the explicit functional form assumed in our work and, arguably, provides more insight into the underlying physiological processes. The method, however, appears to have greater problems with stability, and it was not tested with noisy data. It also required 180 rapid, successive rotations of the camera, which is beyond the capability of most existing systems.

Although we consider in our investigation the application of the method to the heart studies, dynamic imaging methods potentially can be applied to any medical study in which changes in tracer concentration with time are of interest. For example, the renal extraction of tracers such as Tc-99m DTPA [20], and MAG3 [21] and the extraction of $\mathrm{Xe}-133$ in proportion to cerebral blood flow $[22,23]$ are rapid processes in comparison with image acquisition time

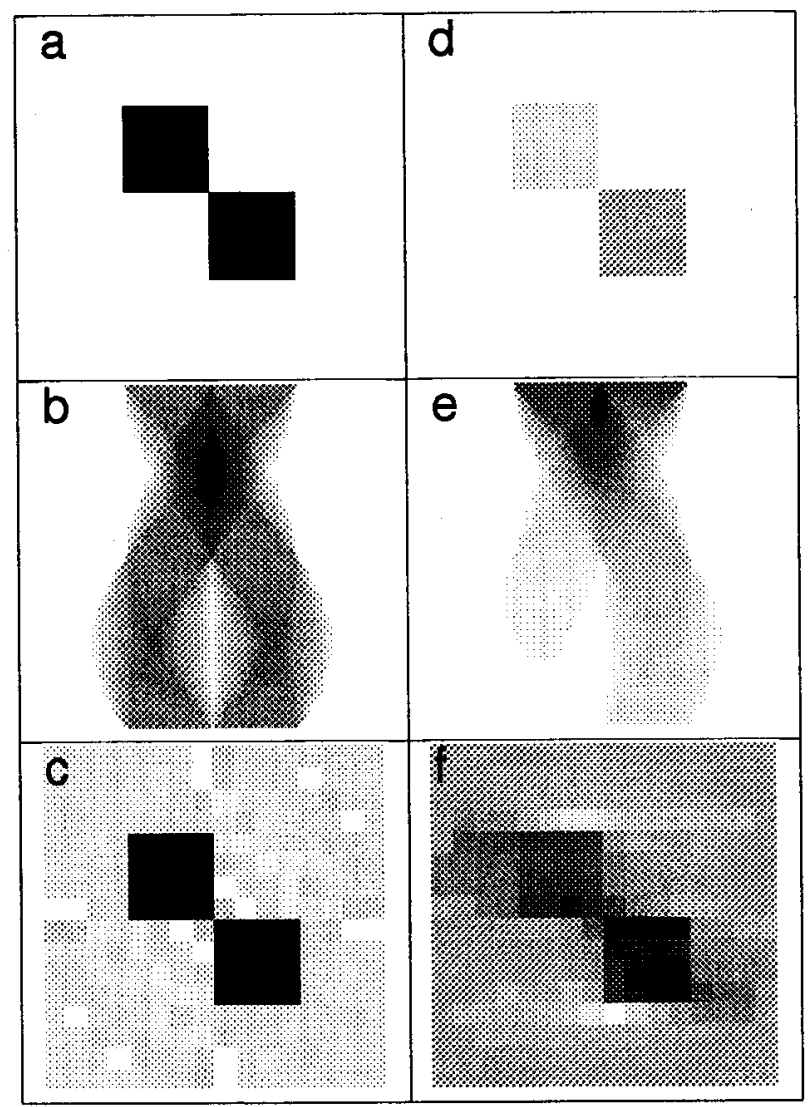

Figure 1: Effects of changing tracer distribution on images reconstructed using filtered backprojection (see text).

and could potentially benefit from dynamic SPECT.

\section{Inconsistent Projections}

As was noted, dynamic studies usually use a sequence of reconstructed images, each intended to represent the spatial distribution of the tracer at some particular moment, and determine kinetic model parameters from the activity values in regions of interest (ROI) in these images. This requires the assumption that the distribution stays constant over the time required to collect the projection set necessary for each image. Tracer distributions that change significantly during the collection time will produce inconsistent data as can be seen in Figure 1.

The simulated object (Figure 1a) consists of two squares of activity. In standard imaging the assumption is made that the activity remains constant in time during acquisition. Figures $1 a, b, c$ illustrate this situation. The same object is shown in Figure 1d, but in this case activity is allowed to decrease exponentially during acquisition, with a half-life of 2.5 minutes in the top square and of 5 minutes in the bottom square. The figure presents the object after 10 minutes. Figures $1 d, e, f$ illustrate the effect of changing activity. Total acquisition time was set to be 20 minutes in both situations. Figures $1 b$ and $1 e$ show the 
sinograms corresponding to the data in Figures 1a and 1d respectively. The images were reconstructed using standard filtered backprojection procedure on a $16 \times 16$ grid. Figure 1c displays the image of the constant activity. Severe artifacts can be seen in Figure 1f which corresponds to the image of the object with changes in activity over the acquisition time.

\section{Parameter Reconstruction}

As discussed in the previous section filtered backprojection does not correctly reconstruct changing activity distributions. To address this problem, we have implemented direct reconstruction of time-activity curve parameters in each image pixel. The major difference between conventional iterative reconstruction methods and methods used in the parameter estimation approach is that the conventional methods construct a system of equations assuming the pixel values are constant, while we assume the activity in the pixel can be modelled as a parameterized function of time and solve the system of equations for the parameters. Consequently, the conventional methods solve a system of linear equations, while we must, in general, solve a system of nonlinear equations.

The data acquired in SPECT studies contain more information than is used. When the activity in the object changes in time, this change is reflected in the projection data and, since each projection is collected at a known time, we can use this additional information. A time dependent function, $x_{i}(t)$, describes the activity in the $i$ th pixel in the object. The following equation can be written for each projection bin by using these functions:

$$
\begin{aligned}
\sum_{i} x_{i}\left(t_{k}\right) a_{i j k} & =d_{j k} \\
j & =1, \ldots, \text { number of bins } \\
k & =1, \ldots, \text { number of projections }
\end{aligned}
$$

where $a_{i j k}$ is the area of the intersection of the $j$ th ray. coming from the angle of the $k$ th projection within the $i$ th region of the heart and $d_{j k}$ is the data collected in the $j$ th bin within the $k$ th projection (which implicitly specifies the time $t_{k}$ ).

The solution to the set of equations can be sought only when some model describing the decay of activity is assumed. In our case, a model proposed for planar studies has been used, where the time-activity curves are described by two exponential components, fast and slow, and a constant.

The number of photons $x$ detected from the $i$ th region of the heart at time $t$ is modelled for fatty acid myocardial viability studies by [17]:

$$
x_{i}(t)=A_{i} e^{-\lambda_{i} t}+B_{i} e^{-\eta_{i} t}+C_{i} .
$$

This problem can be solved in three stages by a curve stripping method, using the fact that the fast component of the biological decay of the fatty acid tracer (related to decay constant $\lambda$ ) in the normal myocardium is much shorter than the slow component (related to $\eta$ ) [17]. Therefore the problem can be split into blocks, each of which can be solved separately. That is, when $t$ is large, the constant $C$ dominates Equation (2), and so $C$ can be determined first in each region of the heart. When $t$ is smaller, the term $B e^{-\eta t}$ dominates $A e^{-\lambda t}$ and so $B, \eta$ can be solved for next. Finally, we can solve for $A, \lambda$ when $t$ is very small. The specific optimal intervals of time $t$ can be determined experimentally. In our implementation we solve this last subproblem, assuming that the data are generated from a single exponential. Thus, our system of equations involves only the variables $A_{i}$ and $\lambda_{i}$.

In order to determine the values of $A$ and $\lambda$ from projection data $d$ (which includes both the usual spatial information and the time the data was collected) in an ideal noise free setting, we have to solve the following nonlinear system of equations:

$$
\begin{aligned}
\sum_{i} A_{i} e^{-\lambda_{i} t_{k}} a_{i j k} & =d_{j k} \\
j & =1, \ldots, \text { number of bins } \\
k & =1, \ldots, \text { number of projections }
\end{aligned}
$$

Because for a realistic situation the number of equations exceeds the number of variables, the problem is overdetermined, and existence of a solution is not guaranteed. Furthermore, patient data is typically noisy and thus an exact solution to Eq. 3 is usually not achievable. To determine the optimal parameter values, we use a least squares method to minimize the norm of the residual. We have chosen to apply the Levenberg-Marquardt method of nonlinear least squares parameter estimation [25].

In the least squares formulation the goal is to minimize the norm of the residual:

$$
\min \left\{\sum_{j, k}\left(\sum_{i} A_{i} e^{-\lambda_{i} t_{k}} a_{i j k}-d_{j k}\right)^{2}: A_{i}, \lambda_{i} \in R\right\} .
$$

Since the Levenberg-Marquardt method is a Newton-like iterative technique, derivative information is required. The necessary gradient and approximate Hessians of the residual functional in Eq. 4 can be explicitly computed. In fact, the computational complexity of an approximate Hessian evaluation is the same as a single function evaluation and so it is not necessary, nor efficient, to use finite difference approximations for derivatives.

For numerical stability in the Levenberg-Marquardt algorithm, we scale the experimental data by the maximum of the data values, so that all data values are between zero and one. We also scale the data acquisition times to lie between zero and one. After convergence is achieved this procedure is reversed. These two scalings imply that during the computations the $A$ 's and $\lambda$ 's are roughly of unit order. In practice, we have found that this scaling dramatically improves convergence of the algorithm. 
The convergence condition is set to be satisfied when either the residual value of $\mathbf{E q} .4$ is smaller than a preset tolerance, or the difference in successive residuals is small. In our experiments, a tolerance of $10^{-4}$ is considered sufficient for satisfactory parameter estimation in the presence of noisy data. This tolerance level is partially justified by our scaling of the data. Numerical experimentation suggests that running the algorithm for a significantly larger number of steps than this criterion requires, causes the solutions to degenerate with respect to the error measure discussed in Section 4. We found that our stopping criterion has produced good results for the tests we have run, but more investigation of these important issues is needed.

A simple model of attenuation was implemented, both in data generation and in parameter reconstruction. With each image pixel, there was associated a certain attenuation factor depending only on the distance from the center of the pixel to the edge of a surrounding cylinder. (See discussion related to a simulated object 5 below.)

The parameter reconstruction algorithm incorporated three standard nonlinear least squares software packages which utilize various modifications of the LevenbergMarquardt algorithm [26, 27, 28]. All three produced comparable results.

Application of these software packages provides sufficient speed of calculations for the simple models investigated in this study. However, for future applications using more complex models, faster methods will be necessary. A possible algorithmic improvement takes note that the structure of the equations is linear in $A, B, C$ and nonlinear in $\lambda$ and $\eta$. Two methods which take advantage of this structure are described in $[29,30]$. These decrease the number of function evaluations by a factor of eight over conventional nonlinear least squares methods. We plan to incorporate these improvements in a future version of the code.

\section{SPECT Experiments}

In the experiments we used analytically generated data to simulate activity distributions that change in time and space. Since parameters can be easily modified in computer simulations, these were used to determine optimal limiting factors related to data acquisition. The goal of the experiments was to investigate the relationships among total initial activity, the rate of change of activity and the SPECT acquisition protocol. Simulation studies modeled data collection in a single 180 degree rotation of the camera. From these data we determined the parameters of monoexponential functions in pixels on grids of up to 16 by 16 pixels.

Each experiment consisted of the following stages:

1. Geometry (number and configuration of pixels), initial activity $(A)$ and rate constant of changing activity distribution $(\lambda)$ of the simulated object were chosen.
2. The complete set of projections was accumulated by summing the activity in the pixels along projection lines. For object 5 (see below) this procedure included attenuation. Each projection can be characterized by the angle and the time when it was acquired.

3. The number of counts in each projection bin was modified to simulate the effect of statistical noise (Gaussian distribution with variance equal to the number of counts in the bin) which corresponded to the modelled activity.

4. Our direct parameter reconstruction algorithm was applied to recover the parameters $\tilde{A}$ and $\tilde{\lambda}$. In experiments including attenuation, attenuation correction was incorporated into the reconstruction algorithm.

Objects with different geometries and different numbers of pixels were modelled. Each pixel in the object was considered to be an ROI in the heart with its particular initial activity and decay constants. Since it is required that the number of equations exceed the number of unknowns, we ensured that the total number of bins in the projections was greater than twice the number of image pixels. This requirement must be met when the patient data is acquired.

Five types of model objects were investigated. In each experiment, total data acquisition time was set to be 20 minutes. The effect of noise on the accuracy of the parameter reconstruction was studied. For each of the model objects, we generated data corresponding to total initial object activity of 500 through 100,000 counts per second. Initial count rate of 2000 counts per second would correspond to a typical injection of $10 \mathrm{mCi}$ of radiopharmaceutical with $5 \%$ heart uptake and $10^{-4}$ detection efficiency of the camera. For objects 1, 2, 4 and 5 sixteen projections spanning $180^{\circ}$ each with sixteen bins were used. Twenty six projections and twenty six bins were used for object 3 .

\section{Simulated Objects:}

1. Uniform initial activity in a $4 \times 4$ object with identical half lifes of 4 minutes in each pixel.

2. Uniform initial activity in a $8 \times 8$ object with identical half lifes of 4 minutes in each pixel.

3. Uniform initial activity in a $16 \times 16$ object with identical half lifes of 4 minutes in each pixel.

4. Simulated heart model. An $8 \times 8$ object with 20 pixels containing equal initial activities (see Figure 3 ). The four quadrants had half lifes of 4, 6, 8, and 10 minutes.

5. Simulated heart model with attenuation. Tests using object 4 were repeated. The heart-like object was placed in a simulated cylinder of water $(\mu=$ $0.15 \mathrm{~cm}^{-1}$ ) with radius one pixel larger than the heart radius. 

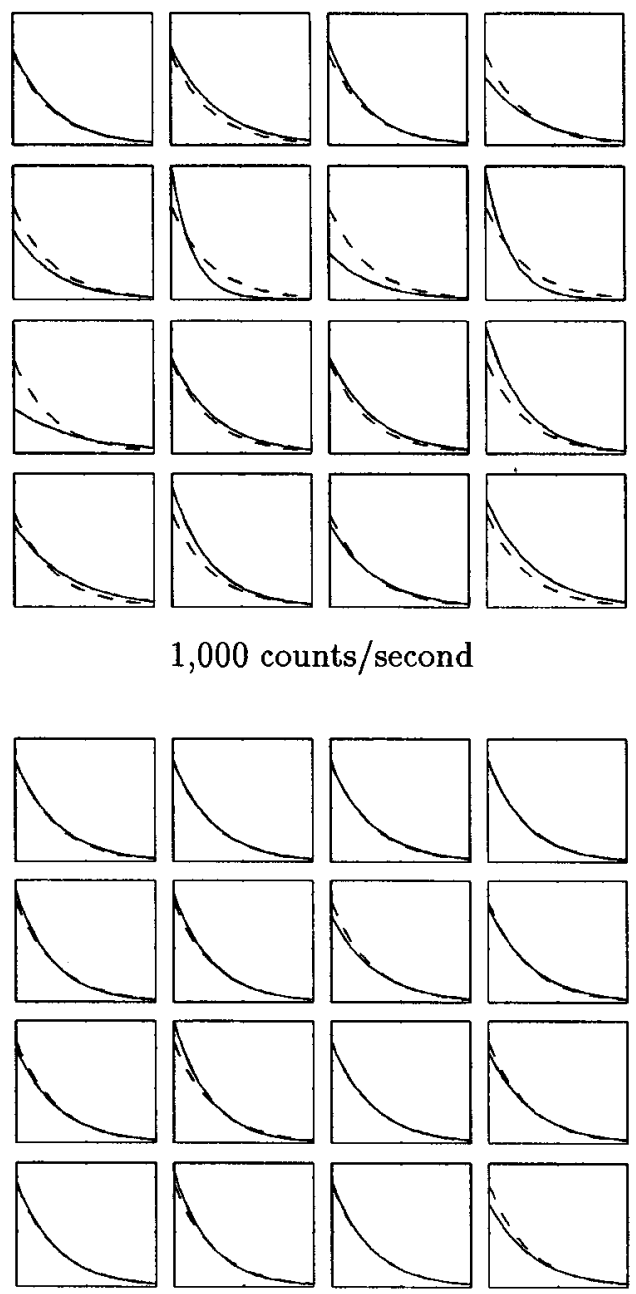

10,000 counts/second

Figure 2: Examples of time-activity curves for each pixel of object 1 . The dashed lines represent the true curves. The solid lines are calculated from the reconstructed parameters.

For each of these data sets 10 independent experiments were run.

In order to assess the accuracy of our reconstructions we needed a measure of the distance between two sets of $A$ 's and $\lambda$ 's, the modelled $\left\{A_{i}, \lambda_{i}\right\}$ and reconstructed $\left\{\tilde{A}_{i}, \tilde{\lambda}_{i}\right\}$. Since we are attempting to match the time activity curves of the true parameters over the data acquisition time interval, we have chosen our measure to be the normalized sum over all reconstruction pixels of the integral of the squares of the difference of the time activity curves:

$$
\text { Error }=\sqrt{\frac{\sum_{i} \int\left(A_{i} e^{-\lambda_{i} t}-\tilde{A}_{i} e^{-\tilde{\lambda}_{i} t}\right)^{2} d t}{\sum_{i} \int\left(A_{i} e^{-\lambda_{i} t}\right)^{2} d t}}
$$

where the integrals are taken over the acquisition times.

For each of the 10 experiments at each object activity level, we computed the reconstructed $\tilde{A}$ 's and $\tilde{\lambda}$ 's and found the mean and variance of the errors.
Table 1: Error for object 1 (uniform activity, $4 \times 4$ pixels).

\begin{tabular}{|c||c|c|}
\hline \multicolumn{1}{|c||}{$\begin{array}{c}\text { total initial } \\
\text { activity }\end{array}$} & \multicolumn{2}{c|}{ Error } \\
\hline counts/second & mean & variance \\
\hline \hline 500 & $8.9 \mathrm{E}-1$ & $2.2 \mathrm{E}+1$ \\
\hline 1000 & $5.7 \mathrm{E}-2$ & $6.1 \mathrm{E}-4$ \\
\hline 5000 & $1.8 \mathrm{E}-2$ & $9.4 \mathrm{E}-5$ \\
\hline 10,000 & $1.1 \mathrm{E}-2$ & $2.1 \mathrm{E}-5$ \\
\hline 20,000 & $5.4 \mathrm{E}-3$ & $5.8 \mathrm{E}-6$ \\
\hline 30,000 & $4.9 \mathrm{E}-3$ & $1.4 \mathrm{E}-5$ \\
\hline 40,000 & $3.4 \mathrm{E}-3$ & $4.2 \mathrm{E}-6$ \\
\hline 100,000 & $1.3 \mathrm{E}-3$ & $2.5 \mathrm{E}-7$ \\
\hline
\end{tabular}

Table 2: Error for object 2 (uniform activity, $8 \times 8$ pixels).

\begin{tabular}{|c||c|c|}
\hline \multicolumn{1}{|c||}{$\begin{array}{c}\text { total initial } \\
\text { activity }\end{array}$} & \multicolumn{2}{c|}{ Error } \\
\hline counts/second & mean & variance \\
\hline \hline 500 & 1.0 & $2.5 \mathrm{E}+1$ \\
\hline 1000 & $6.0 \mathrm{E}-1$ & $2.6 \mathrm{E}-1$ \\
\hline 5000 & $1.3 \mathrm{E}-1$ & $9.9 \mathrm{E}-4$ \\
\hline 10,000 & $8.4 \mathrm{E}-2$ & $4.1 \mathrm{E}-4$ \\
\hline 20,000 & $7.6 \mathrm{E}-2$ & $5.0 \mathrm{E}-4$ \\
\hline 30,000 & $9.0 \mathrm{E}-2$ & $2.6 \mathrm{E}-3$ \\
\hline 40,000 & $6.6 \mathrm{E}-2$ & $6.7 \mathrm{E}-4$ \\
\hline 100,000 & $5.6 \mathrm{E}-2$ & $9.4 \mathrm{E}-4$ \\
\hline
\end{tabular}

\section{Results}

The results of experiments using simulated objects 1 and 2 are presented in Tables 1 and 2 . The mean and variance of the cumulative error (eqn 5) of the distribution are calculated from 10 statistically independent experiments. As expected, the quality of parameter recovery depends on the activity in the object. A threshold activity can be identified above which substantial further improvements are not achieved. A large drop in the value of the variance is an indication that the threshold has been reached. For example, in Table 1 the variance decreases by a factor of almost 100000 between the initial activity levels of 500 and 1000 counts per second.

This analysis is illustrated in Figure 2, where the timeactivity curves of the simulated object 1 , with initial total activity of 1000 and 10000 counts/second, are displayed. The dashed lines represent the true object time-activity curves and the reconstructed curves are plotted as solid lines. When the initial activity in the object was low the statistical noise decreased the accuracy of the reconstruction, which corresponds to a situation illustrated in the top part of Figure 2, where the quality of parameter re- 
Table 3: Error for object 4 (heart-like, no attenuation).

\begin{tabular}{|c||c|c|}
\hline \multicolumn{1}{|c||}{$\begin{array}{c}\text { total initial } \\
\text { activity }\end{array}$} & \multicolumn{2}{c|}{ Error } \\
\hline counts/second & mean & variance \\
\hline \hline 500 & $1.2 \mathrm{E}-1$ & $2.2 \mathrm{E}-3$ \\
\hline 1000 & $5.5 \mathrm{E}-2$ & $6.9 \mathrm{E}-4$ \\
\hline 5000 & $1.2 \mathrm{E}-2$ & $2.7 \mathrm{E}-5$ \\
\hline 10,000 & $5.6 \mathrm{E}-3$ & $1.3 \mathrm{E}-5$ \\
\hline 20,000 & $2.1 \mathrm{E}-3$ & $1.1 \mathrm{E}-6$ \\
\hline 30,000 & $1.7 \mathrm{E}-3$ & $3.5 \mathrm{E}-6$ \\
\hline 40,000 & $1.5 \mathrm{E}-3$ & $4.5 \mathrm{E}-7$ \\
\hline 100,000 & $6.0 \mathrm{E}-4$ & $5.3 \mathrm{E}-8$ \\
\hline
\end{tabular}

Table 4: Error for object 5 (heart-like, with attenuation).

\begin{tabular}{|c||c|c|}
\hline \multicolumn{1}{|c||}{$\begin{array}{c}\text { total initial } \\
\text { activity }\end{array}$} & \multicolumn{2}{c|}{ Error } \\
\hline counts/second & mean & variance \\
\hline \hline 500 & $3.1 \mathrm{E}-1$ & $3.3 \mathrm{E}-2$ \\
\hline 1000 & $2.0 \mathrm{E}-1$ & $1.5 \mathrm{E}-2$ \\
\hline 5000 & $3.8 \mathrm{E}-2$ & $6.3 \mathrm{E}-4$ \\
\hline 10,000 & $2.5 \mathrm{E}-2$ & $1.1 \mathrm{E}-4$ \\
\hline 20,000 & $1.7 \mathrm{E}-2$ & $2.6 \mathrm{E}-5$ \\
\hline 30,000 & $1.4 \mathrm{E}-2$ & $3.1 \mathrm{E}-5$ \\
\hline 40,000 & $1.2 \mathrm{E}-2$ & $2.3 \mathrm{E}-5$ \\
\hline 100,000 & $8.9 \mathrm{E}-3$ & $1.4 \mathrm{E}-5$ \\
\hline
\end{tabular}

covery is inconsistent in the pixels. At the higher activity the parameter recovery is much better.

It should be noted that if the initial activity is distributed over a larger number of object pixels, the threshold activity is higher. Experiments using object $3(16 \times 16$ pixels) yielded similar results with slightly higher threshold levels. The conclusion which can be drawn from this test is that when a patient study is planned, it will be necessary to determine the appropriate threshold levels.

Results of the analysis of object 4 (no attenuation) and object 5 (with attenuation) are presented Tables 3 and 4 . This shape is more complex, with an area of zero activity in the center and four quadrants with different values of $\lambda$. In this case the recovery of parameters is better than for object 2 , which is also simulated using an $8 \times 8$ grid of pixels. This is due to the fact that the activity in the heartlike model is only distributed among 20 pixels rather than 64 pixels and the time constants used here were on average longer than in the other models. Thus, the resulting total number of counts per pixel is higher for the same initial activity.

Figure 3 illustrates the effect of errors in reconstructed parameters for object 4 (with no attenuation) with a total initial activity of 10,000 counts/second. The first column
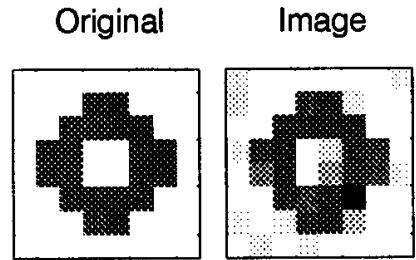

time $=1$
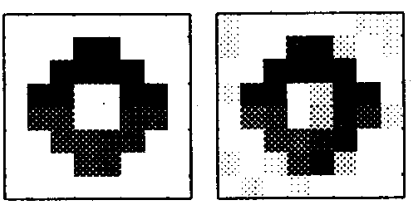

time $=5$
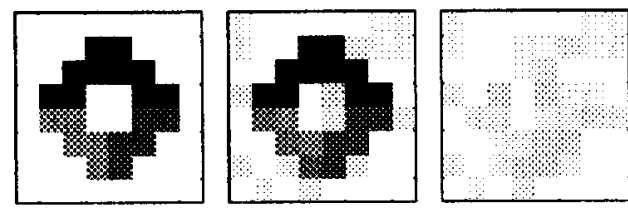

time $=10$
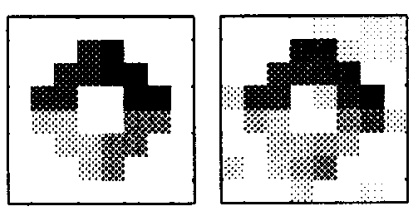

time $=20$


Difference
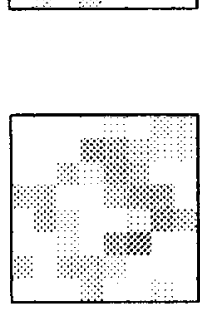

Figure 3: Simulated heart-like object with initial activity of 10,000 counts/second (see text).

of the figure displays the true object activity at different times during the acquisition, calculated using the true $A$ and $\lambda$ for each pixel. The images in the second column are created for the same times using the reconstructed parameters, $\tilde{A}$ and $\tilde{\lambda}$. The difference image in the third column shows the error due to noise induced differences in the $\tilde{A}$ 's and $\tilde{\lambda}$ 's.

The results of experiments for the heart-like object with attenuation are summarized in Table 4 . There is only a slight decrease in the accuracy of parameter reconstruction when compared to the same object without attenuation. This difference may be explained by the decrease in the total number of counts accumulated during acquisition due to the attenuation.

All of the tabulated results are summarized in Figure 4. Total number of counts collected during acquisition would, perhaps, be a more revealing test parameter than the initial object activity, however, this information is generally not available a priori before the study. In this respect, the heart-like object had a greater total number of counts acquired; thus the threshold level for good parameter recovery is lower. 


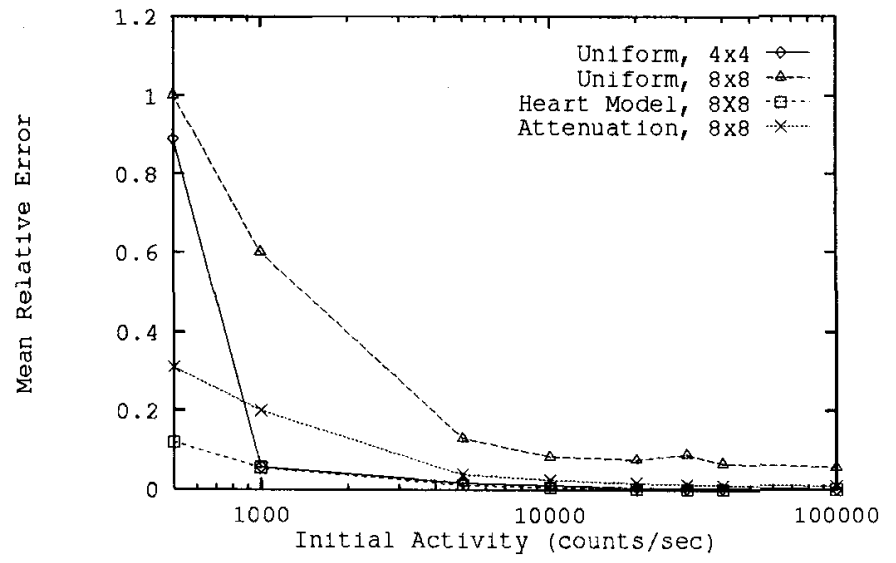

Figure 4: Relative error in parameter reconstruction as a function of total initial activity.

Time for parameter reconstruction typically ranged from about 10 seconds for the $4 \times 4$ object to about 2 hours for the $16 \times 16$ object, independent of the number of counts. The calculations were performed an SGI Indigo R4000 computer.

\section{Conclusions}

We have demonstrated that the parameters of timeactivity curves for a simple model can be recovered directly from the projection data, without reconstructing a time sequence of images. We have developed and tested an iterative method that combines time and spatial information to determine these parameters. This new method will allow dynamic studies to be performed on standard rotating SPECT cameras with only one or two heads.

A series of preliminary experiments was performed to investigate the count rate requirements of the method. Simulated data for different object geometries and total initial activity varying from 500 to 100,000 counts/second were used in the studies. The accuracy of the parameter recovery was good for object activities similar to those expected for myocardial viability studies. The effect of attenuation was included in the studies. The conclusion is that when the appropriate attenuation correction is applied the recovery of the time-activity curve parameters is not influenced by this effect, provided the sufficient number of counts are recorded.

More investigation is required to determine the optimum acquisition protocols for a particular activity and half-life. The performance of the numerical methods, including the speed of convergence and stopping criteria, should be investigated. Other models describing the change of activity, including the full equation 2 should be studied.

\section{References}

[1] J Tillisch, R Brunken, R Marshall, M Schwaiger, M Mandelkern, M Phelps, H Schelbert. Reversibility of cardiac wall-motion abnormalities predicted by positron tomography. N Engl J Med. $1986 ; 314: 884-888$.

[2] GL Murray, NC Schad, HL Magill, R Vander Zwaag. Myocardial viability assessment with dynamic low-dose iodine-123phenylpentanoic acid metabolic imaging: Comparison with myocardial biopsy and reinjection SPECT thallium after myocardial infarction. J Nucl Med 1994;35:43S-48S.

[3] MP Hudon, DM Lyster, WRE Jamieson, AK Qayumi, M Keiss, LJ Rosado, AP Autor, C Sartori, H Dougan, J Van Den Broek. Efficacy of $\left({ }^{123} \mathrm{I}\right)$-iodophenyl pentadecanoic acid (IPPA) in assessment myocardial metabolism in a model of reversible global ischemia. Eur J Nucl Med. 1988;14:594-599.

[4] ME Phelps, JC Mazziotta, Schelbert, eds. Positron Emission Tomography and Autoradiography: Principles and Applications in Brain and Heart. New York, Raven Press, 1986.

[5] BM Mazoyer, RH Huesman, TF Budinger, BL Knittel. Dynamic PET Data Analysis. J Comput Assist Tomogr 1986;10:645-653.

[6] B Mickey, S Vorstrup B Voldby, H Lindewald, A Harmsen, NA Lassen. Serial measurement of regional cerebral blood flow in patients with $\mathrm{SAH}$ using ${ }^{133} \mathrm{Xe}$ inhalation and emission computerized tomography. J Neurosurg 1984;60:916-922.

[7] NA Lassen, B Sperling. ${ }^{99 m}$ Tc-bicisate reliably images CBF in chronic brain diseases but fails to show reflow hyperemia in subacute stroke: Report of a multicenter trial of 105 cases comparing ${ }^{133} \mathrm{Xe}$ and ${ }^{99 m}$ Tc-bicisate (ECD, Neurolite) measured by SPECT on the same day. J Cereb Blood Flow Metab 1994;14(Suppl. 1):S44-S48.

[8] CL Hansen. Preliminary report on an ongoing phase I/II dose range, safety and efficacy study of iodine-123-phenylpentanoic acid for the identification of viable myocardium. J Nucl Med $1994 ; 35: 38 \mathrm{~S}-42 \mathrm{~S}$.

[9] K Nakajima, J Taki, H Bunko, M Matsudaira, A Muramori, I Matsunari, K Hisada, T Ichihara. Dynamic acquisition with a three-headed SPECT system: application to technetium $99 \mathrm{~m}-$ SQ30217 myocardial imaging. J Nucl Med 1991;32:1273-1277.

[10] AM Smith, GT Gullberg. Dynamic cardiac SPECT computer simulations for teboroxime kinetics. IEEE Trans Nucl Sci 1994;41:1626-1633.

[11] AM Smith, GT Gullberg, PE Christian, FL Datz. Kinetic modeling of teboroxime using dynamic SPECT imaging of a canine model. J Nucl Med 1994;35:484-495.

[12] RE Carson, $K$ Lange. The EM parametric image reconstruction algorithm. J Am Stat Assoc. 1985;80:20-22.

[13] DL Snyder. Parameter estimation for dynamic studies in emission tomography systems having list mode data. IEEE Trans Nucl Sci. 1984;31:925-931.

[14] JM Ollinger, DL Snyder. An evaluation of an improved method for computing histograms in dynamic tracer studies using positron emission tomography. IEEE Trans Nucl Sci. $1986 ; 33: 435-438$.

[15] JM Ollinger. Estimation algorithms for dynamic tracer studies using positron emission tomography. IEEE Trans Med Imag. 1987;6:115-125.

[16] P Chiao, WL Rogers, NH Clinthorne, JA Fessler, AO Hero. Model-based estimation for dynamic parameters using ECT. IEEE Trans Med Imag. 1994;13:217-226. 
[17] MPJ Hudon, DM Lyster, WRE Jamieson, AK Qayumi, C Sartori, and HA Dougan. The metabolism of $\left({ }^{123} \mathrm{I}\right)$-iodophenyl pentadecanoic acid in a surgically induced canine model of regional ischemia. Eur J Nucl Med. 1990;16:199-204.

[18] MJ van Eenige, FC Visser, CMB Duwel, PD Bezemer, G Westera, AJP Karreman, JP Roos. Analysis of myocardial timeactivity curves of ${ }^{123}$ I-heptadecanoic acid. I. Curve Fitting. Nucl-Med. 1987;26:241-7.

[19] Zeng GL, Gullberg GT. Estimating kinetic parameters directly from projection measurements. To appear in Conference record of the 1994 IEEE Nuclear Science Symposium and Medical Imaging Conference.

[20] Y Takaki, A Kojima, A Tsuji, R Nakashima, S Tomiguchi, M Takahash. Quantification of renal uptake of Technetium-99mDTPA using planar scintigraphy: A technique that considers organ volume. J Nucl Med. 1993;34:1184-1189.

[21] B Bubeck, W Brandan, E Weber, T Kälble, N Parekh, P Georgi. Pharmacokinetics of Technetium-99-m-MAG3 in humans. J Nucl Med. 1990;31:1285-1293.

[22] P Celsis, T Goldman, L Henriksen, NA Lassen. A method for calculating regional cerebral blood flow from emission computed tomography of inert gas concentrations. J Comput Assist Tomogr. 1981;5:641-645.

[23] MD Devous Jr, JK Payne, JL Lowe, RF Leroy. Comparison of Technetium-99-m-ECD to Xenon-133 SPECT in normal controls and in patients with mild to moderate regional cerebral blood flow abnormalities. J Nucl Med. 1993;34:754-761.

[24] D Huglo, M Rousseaux, D Leys, P Fialdes and M Steinling. Regional cerebral blood flow imaging: A quantitative comparison of ${ }^{99 m}$ Tc-bisicate with ${ }^{133}$ Xe using single photon emission computed tomography. J Cereb Blood Flow Metab 1994;14(Suppl. 1):S76-S83.

[25] D Marquardt.An algorithm for least-squares estimation of nonlinear parameters. SIAM J Appl Math. 1963;11:431-441.

[26] PT Boggs, RH Byrd, JE Rogers, and RB Schnabel. User's Reference Guide for ODRPACK Version 2.01. NISTIR 924832,1992 .

[27] Matlab User's Guide. Math Works Inc. Natick MA, 1992.

[28] J Moné, B Garbow and K Hillstrom. Minpack. Argonne Nat'l Lab.

[29] L Kaufman. A variable projection method for solving separable nonlinear least squares problems. BIT. 1975;49-57.

[30] GH Golub and V Pereyra. The differentiation of pseudo-inverses and nonlinear least squares problems whose variables separate. SIAM J of Numer Anal. 1973;10 \# 2:413-432. 